\title{
Design and Debugging of GIS fault fast positioning system based on PVDF piezoelectric film sensor
}

\author{
YANG Zhaoguang ${ }^{1, a^{*}}$, LI Zhixin ${ }^{2, b}$, YANG Junting ${ }^{1, \mathrm{c}}$, WEN Dingjun ${ }^{1, \mathrm{~d}}$ \\ ${ }^{1}$ Gansu Electric Power Research Institute ,Lanzhou, China; \\ 2 Training Center of Gansu Electric Power Company, ,Lanzhou, China \\ a307060147@qq.com, b595383313@qq.com, 'oleole@aliyun.com, d1379189837@qq.com
}

\begin{abstract}
Keywords: GIS; Flashover Locationing; PVDF; Sensor
Abstract. This paper introduces an acoustic emission sensor using PVDF Piezoelectric film which is convenient to use in the field. In order to verify the reliability of GIS fault fast positioning system based on PVDF piezoelectric film sensor, four different kinds of typical defects were set, and the practical research on GIS flashover positioning was carried out. The result shows that by comparing sound intensity of different gas rooms, GIS fault fast positioning system based on PVDF piezoelectric film sensor, the fault location can be found accurately.
\end{abstract}

\section{Introduction}

In AC withstand voltage test of GIS, restricted by field condition, The location of the GIS flashover and puncture was mainly judged by human ear or ultrasonic locator and ultra high frequency locator. Ultrasonic locator and ultra high frequency locator were mostly limited by the number of sensor channels, meanwhile, the cost of the instrument was high and inconvenient to carry. Therefore, they can not be widely used in the field. As a result, it is urgent to study a flashover and breakdown of the gas chamber fault location system which can locate accurately and is practical, low power consumption, portable, and with signal displaying.

Compared with the conventional piezoelectric materials, PVDF piezoelectric film materials have many advantages such as: wide frequency response, high mechanical performance, light weight, good flexibility, less susceptible to pollution by water and chemicals, strong plasticity and so on, have been widely used in various industry fields[1-3]. Extensive applications showed that the sensor made of this material has high sensitivity, overload resistance, good anti-interference simple operation, small volume, and light weight[4,5]. This paper designs a kind of flashover positioning system based on PVDF piezoelectric film sensor, which can quickly and accurately locate the discharge position by collecting and comparing the intensity of the acoustic signal from different sensor when the breakdown or flashover happened.

\section{Design ideas}

When the GIS flashover occurs, the sound waves propagate along the metal case, with reflection and refraction at the interface between the metal and the insulator. Sound waves pass from the air chamber where flashover occured through insulator to the adjacent gas chamber, the attenuation of acoustic vibration is very obvious[8-11]. Therefore, the GIS fault gas chamber can be quickly located by the GIS fault location system based on the PVDF piezoelectric film sensor. The working diagram of GIS flashover breakdown positioning system is as follows:

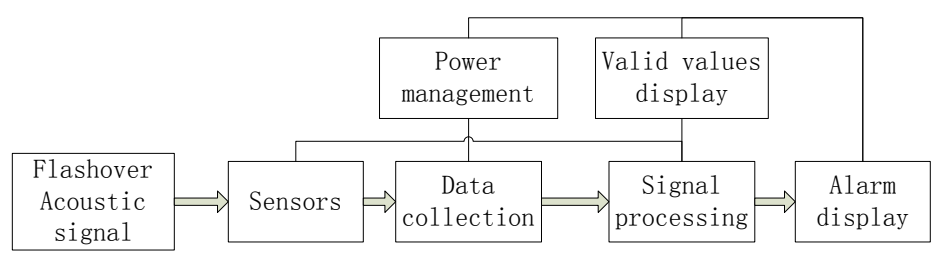

Fig. 1 GIS flashover locator principle diagram 


\section{Sensor performance test}

Using the signal of step function form sent by the pencil core breaking to simulate the acoustic emission source, key performance parameters such as frequency response range and sensitivity can be detected. In the acoustic emission experiments, $\Phi 0.5 \mathrm{~mm}$ pencil core and $800 \mathrm{~mm} * 800 \mathrm{~mm} * 3$ $\mathrm{mm}$ aluminum plate were used. The principle of the experiment is shown in figure 2.]

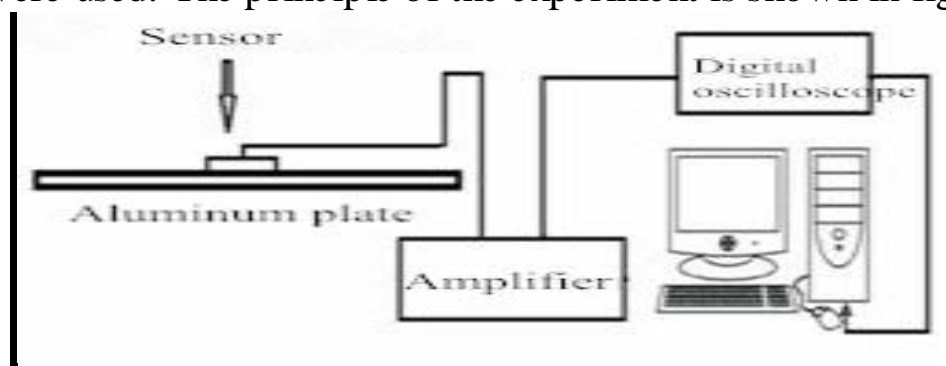

Fig.2 The principle of test system

At $20 \mathrm{~mm}$ distance far from sensor, the pencil core was broken. The sensor received the signal, the time domain waveforms and frequency domain waveforms are shown in figure 3 and figure 4 separately.

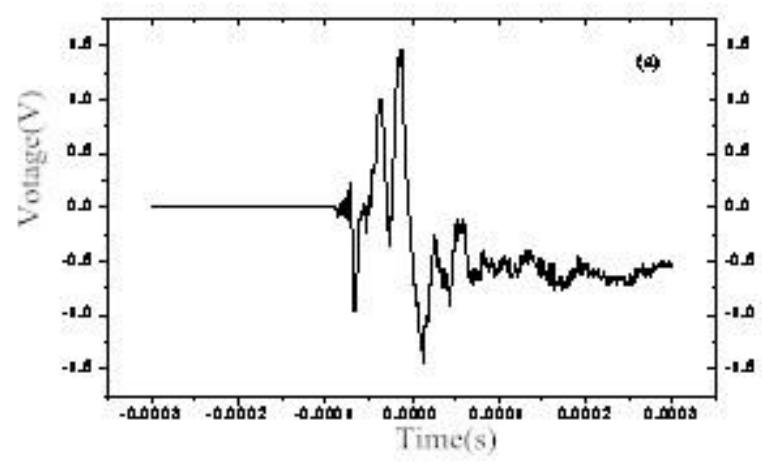

Fig.3 The time domain signal that sensor 1 received

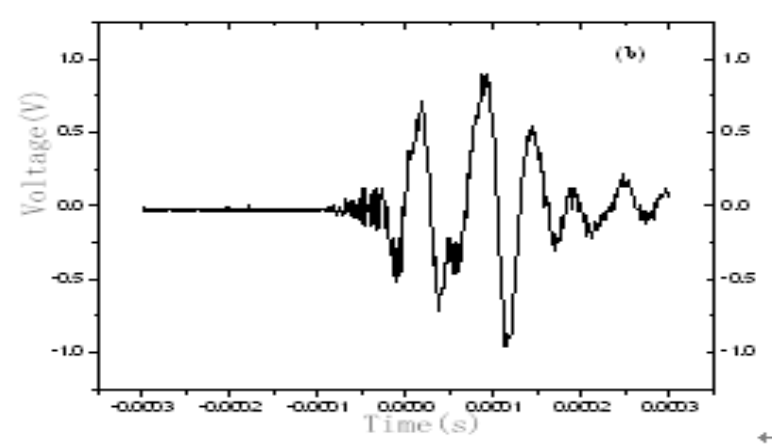

Fig.5 The time domain signal that sensor 2received

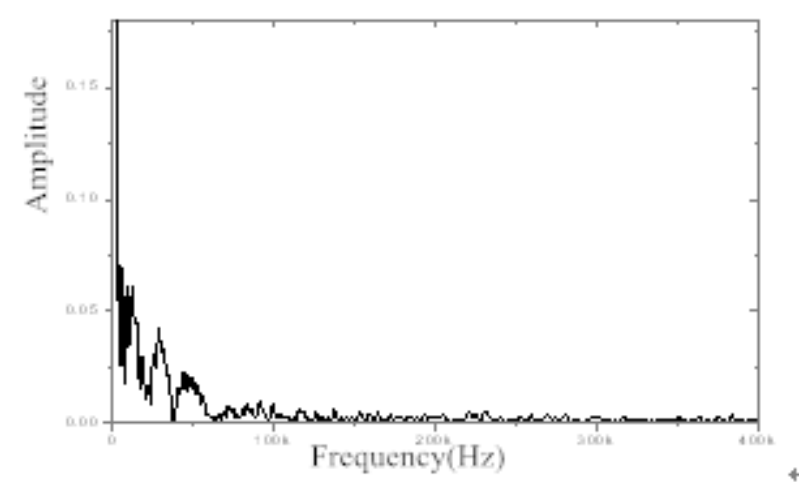

Fig.4 The frequency domain signal that sensor 2 received

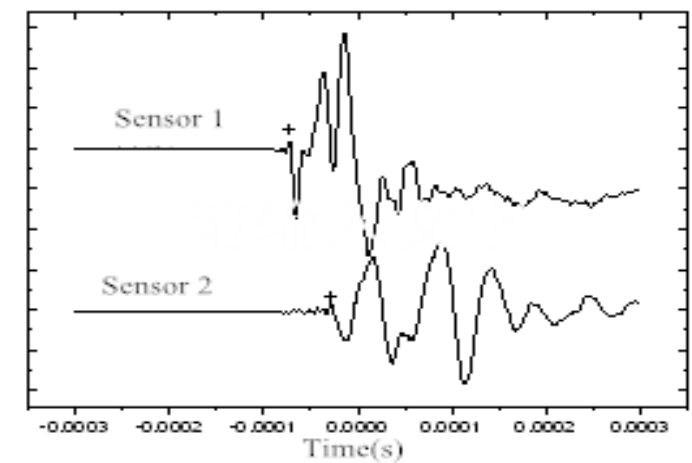

Fig.6 The comparison of time domain signal that two sensors received

Figure 3 shows that the peak voltage of signal obtained by the se nsor reached $1.48 \mathrm{~V}$, which means the sensor has a high sensitivity. Figure 4 shows that the signal frequency of the the pencil core fracture is distributed in the range of $0-100 \mathrm{kHz}$, which indicates that PVDF piezoelectric film sensor has a wide frequency response. In the experiment, two sensors were installed on the aluminum plate and kept a different distance from the pencil core fracture. Sensor 1 is $20 \mathrm{~mm}$ far from the pencil core fracture, and sensor 2 is located on the straight line of sensor 1 and the pencil core, it is $20 \mathrm{~mm}$ from sensor $1,40 \mathrm{~mm}$ from the pencil core. Through the comparison of figure 3 and figure 5 , with the increase of signal transmission distance, the peak of signal intensity attenuates $17 \%$, from $1.48 \mathrm{~V}$ to $0.9 \mathrm{~V}$. This shows that the sensor has a good resolution to acoustic attenuation.

Compare the time cost that two sensors received the first wave, it can be seen that sensor 1 which is closer to the signal source received the signal first, the time is $4.3 \times 10-5 \mathrm{~s}$ earlier than sensor 2 . This can calculate the speed of acoustic wave spreading in aluminum which is approximately $4652 \mathrm{~m} / \mathrm{s}$. 
This is very close to $5134 \mathrm{~m} / \mathrm{s}$ in the literature. The error mainly comes from measurement of time. The results show that PVDF piezoelectric film sensor has characteristics such as high sensitivity, high resolution, wide frequency response range and easy to use.

\section{Practical research of GIS fault fast positioning system under the different defect models}

Four kinds of typical defect models which may cause flashover in GIS AC withstand voltage test were set to verify the reliability of GIS flashover fault location system. They are: metal tip discharge on cond uctive pole(Model1), metal tip discharge on the inner surface (Mode12), Suspended electric potential discharge(Mode13) and insulator filth discharge(Model4).

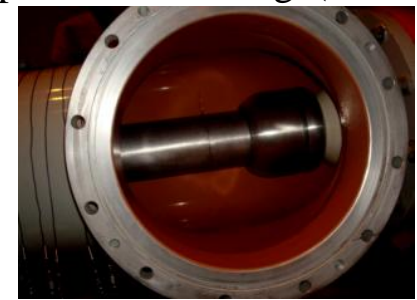

Fig. 7 Model 1

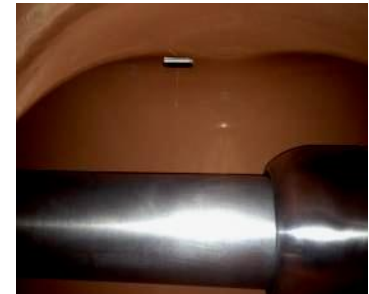

Fig. 8 Model 2

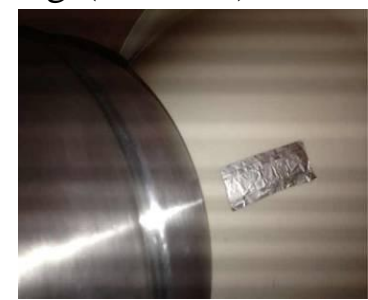

Fig. 9 Model 3

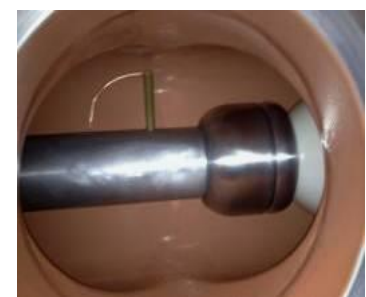

Fig. 10 Model 4

The sound intensity of the flashover under different defects was measured, the result was shown in figure 11-figure 14.

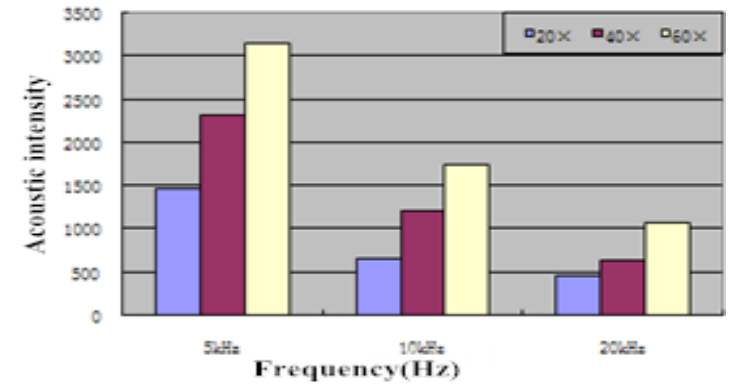

Fig 11 Sound intensity distribution of model 1

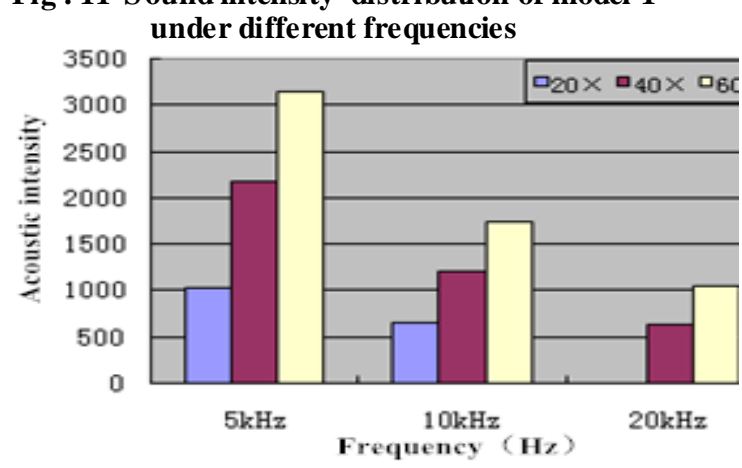

Fig . 13 Sound intensity distribution of model 3 under different frequencies

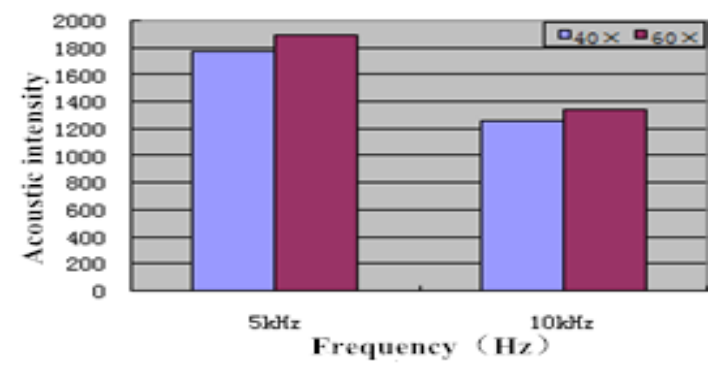

Fig . 12 Sound intensity distribution of model 2 under different frequencies

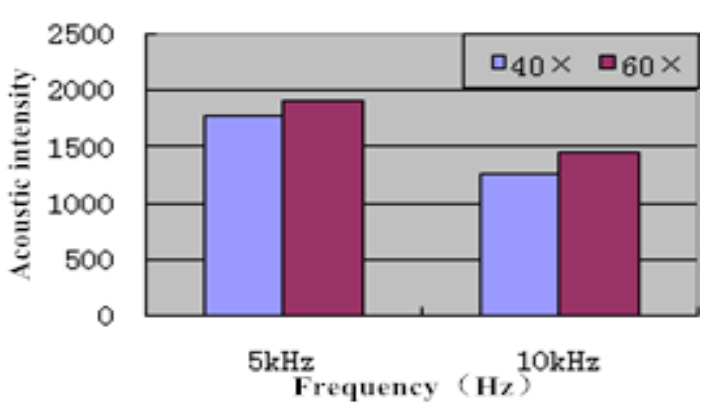

Fig . 14 Sound intensity distribution of model 4 under different frequencies

Experimental data show that when flashover breakdown occurs, the acoustic intensity signals are mainly in the low frequency range. When flashover occurs, to the different defect discharge model, the sound intensity signals are of different frequency ranges. However, in all the test results, low frequency components are relatively rich, they are mainly concentrated in between 5 and $20 \mathrm{kHz}$.

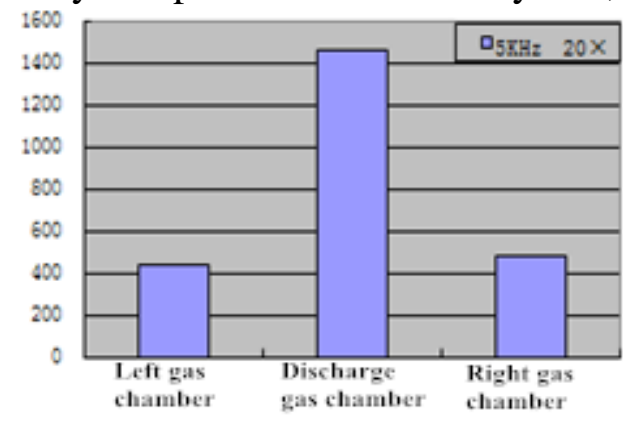

Fig. 15 Sound intensity distribution of the

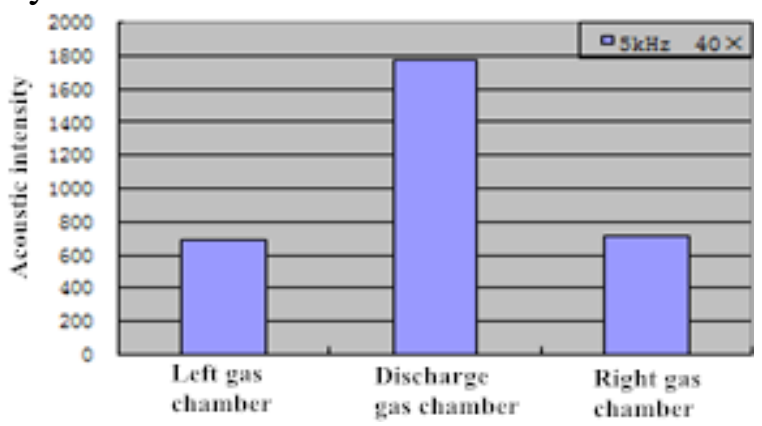

Fig . 16 Sound intensity distribution of the 
different gas chamber of model 1

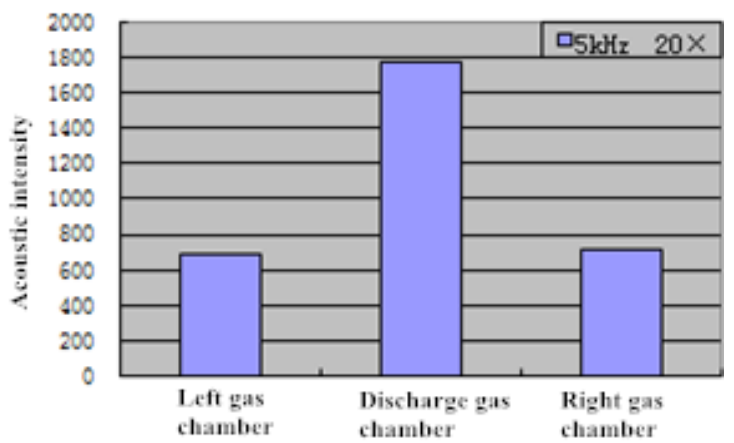

Fig . 17 The sound intensity distribution of the different gas chamber of model 3 different gas chamber of model 2

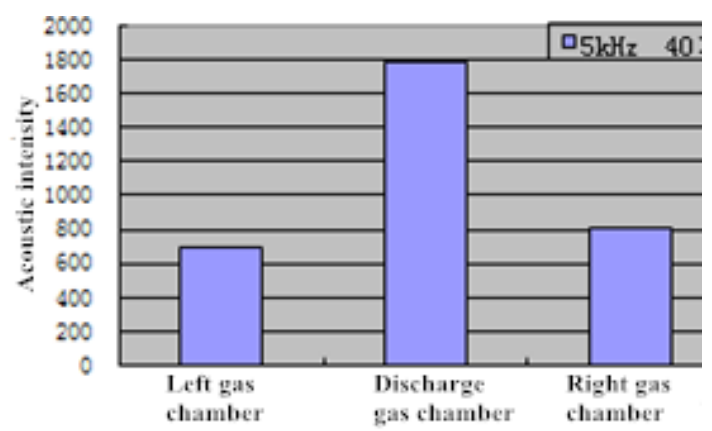

Fig. 18 The sound intensity distribution of the different gas chamber of model 4

Test results shows that by measuring acoustic intensity signal using GIS fault fast positioning system based on PVDF piezoelectric film sensor, it is found that acoustic intensity signal of the discharge gas chamber is obviously different from that of the adjacent gas chamber. The acoustic intensity signal of the discharge gas room is 2-3 times larger of that of the adjacent gas chamber.

\section{Conclusions}

GIS fault fast positioning system based on PVDF piezoelectric film sensor could locate the fault accurately by comparing the acoustic intensity of each gas chamber, avoiding cumulative damage on GIS insulation caused by repeated AC withstand voltage test. Its sensitivity and resolution are high, the frequency response range is wide, the anti-jamming ability is strong, and thus it is convenient in field use.

\section{Reference}

[1] YANG Zhaoguang, ZHANG Taoyun, WEN Dingjun, et al. Development of acoustic emission sensor based on the 0-3 PZT/P(VDF-TFE) piezoelectric composite[J]. Electronic Components and Materials,2014,33(6):69-71.

[2] SMITH W A. The role of piezocomposites in ultrasonic transducers [C]//Proceeding of the 1989 IEEE Ultrasonic Symposium. Montreal,Canada: IEEE, 1989: 755-766

[3] HE Cunfu, GE Haiyan, JIAO Jingpin,el. PVDF Comb Transducer and its Application in Ultrasonic Guided Wave Testing [J]. Nondestructive Testing, 2007,1,16-21

[4] YUAN Renshu, SUI Shiqun. Dynamic Response of PVDF Thin Film under Pulse Pressure[J]. Instrument Technique and Sensor ,2011,8:4-6.

[5] KAISER J. Untersuchungen über das Auftreten Gerauschen beim

[6] Zugversuch [D]. München: Technische Hochschule, 1953.

[7] GARNER G M, SHRROCKS N M, WHATMORE R W, et al. 0-3 Piezoelectric Composites for Large Area Hydrophones [J]. Ferroelectrics,1989, 93: 169-176.

[8] NEWNHAM R E, SAFARI A, GINIEWICES J, et al. Compositepiezoelectric sensors [J]. Ferroelectrics, 1984, 60:15-21.

[9] MILLER R K. Nondestructive Testing Handbook, 2nd edition: Acoustic Emission Testing [M]. Columbus OH: American Society for Nondestructive Testing, 1987: 74-76

[10] WANG Qingfeng,WU Bin,JIAO Jingpin. The Design Method and Application of PVDF Aacoustic Emission Sensor[J].Instrument Technique and Sensor ,2006,11:23-27

[11] PEREZ RUIZ S J, ALCANTARA INIESTA S, HERNANDEZ P R, et al.Sound speed resolved by photoacoustic technique [J]. Rev Mex Fis, 2007,53(3): 213. 